\title{
Effects of sealant, viscosity, and bonding agents on microleakage of fissure sealants: An in vitro study
}

\author{
Maryam Mehrabkhani ${ }^{1}$, Fatemeh Mazhari ${ }^{2}$, Samaneh Sadeghi ${ }^{3}$, Masoumeh Ebrahimi ${ }^{4}$
}

Correspondence: Dr. Fatemeh Mazhari

Email: mazharif@mums.ac.ir

\begin{abstract}
'Department of Pediatric Dentistry, Dental Research Center, Faculty of Dentistry, Mashhad University of Medical Sciences, Mashhad, Iran, 2Department of Pediatric Dentistry, Dental Material Research Center, Faculty of Dentistry, Mashhad University of Medical Sciences, Mashhad, Iran, ${ }^{3}$ Department of Orthodontic, School of Dentistry, Kerman University of Medical Sciences, Kerman, Iran, ${ }^{4}$ Department of Pediatric Dentistry, Oral and Maxillofacial Diseases Research Center, Faculty of Dentistry, Mashhad University of Medical Sciences, Mashhad, Iran
\end{abstract}

\section{ABSTRACT}

Objective: The aim of this study was to evaluate the effects of enamel or dentin bonding agent (DBA) and sealant viscosity on sealant microleakage. Materials and Methods: Sixty extracted human premolars were randomly divided into two equal groups (based on sealant viscosity) and each group was divided into three subgroups of 10 teeth. Group 1 (low viscosity sealant, Seal-Rite, Pulpdent, USA with 7.7\% filler): Prophylaxis, enameloplasty, etching of occlusal surfaces with $38 \%$ of phosphoric acid gel, rinsing and drying, followed by (1) enamel bonding agent (EBA) (Margin Bond, Coltène/Whaledent AG) or (2) DBA (Excite, Ivoclar Vivadent AG, Liechtenstein) or (3) no bonding (NB) prior to sealant application. In Group 2, similar procedures were performed except for applying a high viscosity sealant (Seal-Rite, Pulpdent, The USA with $34.4 \%$ filler). Specimens were thermocycled and then immersed in a $0.5 \%$ basic fuchsine solution for $24 \mathrm{~h}$ next, buccolingual slices of samples were scored under a stereomicroscope. The Kruskal-Wallis and Mann-Whitney U-tests were used for data analysis. Results: There was no significant difference between DBA, EBA, and NB subgroups in the microleakage scores in both groups. Low viscosity sealant had a lower microleakage than the high viscosity sealant in both DBA $(P=0.002)$ and NB $(P=0.041)$ subgroups. Conclusion: The results indicated that the use of low viscosity sealant reduced the microleakage of pit and fissure sealants. However, the use of a bonding agent before sealant placement didn't affect the microleakage.

Key words: Dentin bonding agent, enamel bonding agent, microleakage, sealant viscosity

\section{INTRODUCTION}

During the past several decades, caries incidence in pediatric and adolescent groups has dramatically decreased, especially with respect to smooth surface lesions. However, despite this improvement, dental caries are particularly common on the surfaces with pits and fissures. To overcome this problem sealant - a material placed into caries-susceptible pits and fissures - is used to prevent the access of cariogenic bacteria to the source of nutrients. Thus, pit and fissure sealant could be a safe and effective way to prevent dental caries. ${ }^{[1]}$ However, failure to use sealants correctly can cause leakage with partial or total loss leading to sealant failure at a rate of $5-10 \%$ years. ${ }^{[2]}$
So far, several studies have been done focusing on improving the success of fissure sealants. ${ }^{[3,4]}$ An important factor to consider in the success of sealants is the prevention of microleakage, the ingress of bacteria and oral fluids into the space between the

This is an open access article distributed under the terms of the Creative Commons Attribution-NonCommercial-ShareAlike 3.0 License, which allows others to remix, tweak, and build upon the work non-commercially, as long as the author is credited and the new creations are licensed under the identical terms.

For reprints contact: reprints@medknow.com

How to cite this article: Mehrabkhani M, Mazhari F, Sadeghi S, Ebrahimi M. Effects of sealant, viscosity, and bonding agents on microleakage of fissure sealants: An in vitro study. Eur J Dent 2015;9:558-63.

DOI: $10.4103 / 1305-7456.172631$ 
tooth and restorative material. Since microleakage can result in caries lesion development underneath the sealant, inhibiting marginal leakage is critical for sealant success. It has been shown that the application of the intermediate bonding layer between enamel and sealant after contamination of etched enamel with saliva improves result. ${ }^{[5]}$ Other studies suggest that the application of the bonding agent before sealant improves bond strength and decreases microleakage, ${ }^{[6,7]}$ although some studies have shown no such differences. ${ }^{[8-10]}$

There are two main groups of bonding agents: Enamel bonding agent (EBA) (hydrophobic agent) and dentin bonding agent (DBA) (hydrophilic agent). Given that the pit and fissures of occlusal surfaces consist mostly of enamel, it seems that it is better to use EBA than DBA, but there is no conclusive evidence to support it.

Some investigations have shown that sealant viscosity may adversely affect the success of fissure sealant. ${ }^{[11]}$ However, there are some contraindicatory findings of the effect of sealant viscosity. Some studies have indicated that compared to high viscosity resin, low viscosity sealant material exhibits better marginal adaptation. ${ }^{[12-15]}$ On the other hand, other studies have reported no difference between unfilled and filled sealants regarding microleakage scores. ${ }^{[16]}$

The aim of this study was to evaluate the effect of enamel bonding, dentin bonding, and sealant viscosity on microleakage of fissure sealant.

\section{MATERIALS AND METHODS}

This study was carried out at the Pediatric Department of the Dental School with the permission of the Research and Ethics Committee, School of Dentistry, University of Medical Science (Mashhad, Khorasan, Iran).

In this in vitro study 60 human maxillary premolars extracted for orthodontic reasons were used. All teeth were cleaned and stored in $0.2 \%$ of thymol solution at room temperature. After cleansing with a rubber cup and pumice powder, occlusal fissures were slightly opened using a fissurotomy bur (Fissurotomy ${ }^{\circledR}$ Original - FGSS SS White, Lakewood, NJ USA) on a high-speed handpiece. The teeth were randomly assigned to two equal groups based on the type of sealant viscosity, and each group was divided into three subgroups of 10 teeth.

Group 1 (subgroup 1): The occlusal pit and fissures were etched (Etch Rite, Pulp Dent, USA) for 20 s, and then rinsed for $15 \mathrm{~s}$ and thoroughly air dried with oil-free compressed air to obtain a white, dull and chalky-like appearance. Next, a low filler sealant of $7.7 \% \mathrm{w} / \mathrm{w}$ (Seal-Rite, Pulp Dent, USA) was applied, and light-cured for $40 \mathrm{~s}$.

Group 1 (subgroup 2): The teeth were conditioned similarly to the previous subgroup, but prior to low filler sealant placement, EBA (Margin Bond, Coltène/Whaledent AG) was applied with a hand-held brush, air-thinned after $10 \mathrm{~s}$ and light cured for $20 \mathrm{~s}$.

Group 1 (subgroup 3): The procedure was similar to Group 1 (subgroup 2), except instead of EBA, DBA (Excite, Ivoclar Vivadent AG, Liechtenstein) was applied before sealant placement according to the manufacturer's instructions.

Group 2 and its subgroups went through similar processes, except a high filler sealant of $34.4 \% \mathrm{w} / \mathrm{w}$ (Seal-Rite, Pulp Dent, USA) was used.

Polymerization was performed using a halogen light curing unit (Coltolux 75, Coltene/Whaledent) with an output of $500 \mathrm{~mW} / \mathrm{cm}^{2}$. The light intensity was checked periodically with the digital radiometer (Coltolux ${ }^{\circledast}$, Coltène/Whaledent Inc.). . All specimens were stored in distilled water at $37^{\circ} \mathrm{C}$ for a week, thermocycled for 500 cycles between $5^{\circ} \mathrm{C}( \pm 2)$ and $55^{\circ} \mathrm{C}( \pm 2)$ with a $10 \mathrm{~s}$ dwell time at each temperature. All teeth surfaces, except for the $1 \mathrm{~mm}$ outside the margins of the sealant, were double coated with nail varnish and were immersed in a $0.5 \%$ basic fushin dye solution for $24 \mathrm{~h}$. Following immersion, all teeth were washed under running tap water for $30 \mathrm{~s}$ to remove excess dye solution, and then embedded in epoxy resin and sectioned in the buccolingual direction at mesial and distal pits with a water-cooled diamond disk (Isomat 2000, Buehler, Lake Bluff, IL, USA) to create three sections and four surfaces per tooth, each approximately $1.5 \mathrm{~mm}$ in thickness.

For microleakage evaluation, one trained and blinded examiner scored the dye penetration depth in each section using a stereomicroscope (Wild, Leitz Ltd., Herbage, Switzerland) at $\times 40$. Thus, the degree of microleakage judgment was kept blind.

The scoring system used in the present study was as follows:

- Score 0: No dye penetration

- Score 1: Dye penetration limited to the outer half of the sealant 
- Score 2: Dye penetration to the inner half of the sealant

- Score 3: Dye penetration extends into the bottom of the fissure.

This evaluation scale is the most widely used in previous studies on sealant microleakage. ${ }^{[17]}$ Where scores were different at four surfaces per tooth, the worse score (higher degree of leakage) was referred to as the leakage score.

To determine statistically significant differences in leakage among the subgroups in the two groups (low or high viscosity) separately, nonparametric data were analyzed using the Kruskal-Wallis test. The Mann-Whitney U-test was performed to compare the leakage between the two viscosities at each subgroup separately. All statistical analyses were performed using SPSS version 15.0 for Windows (SPSS Inc., Chicago. IL., USA) at the significance level of $P<0.05$.

\section{RESULTS}

The present study aimed to assess comparatively the microleakage of teeth sealed with high or low filled sealants (Seal-Rite) with and without the application of bonding agents (Exite or Margin Bond).

Hence, 60 teeth (240 surfaces) were evaluated in this study for microleakage. The frequency distribution of microleakage between various groups is shown in Table 1 . At the low viscosity group, EBA subgroup had the maximum microleakage, followed by no bonding (NB) subgroup; and, minimum microleakage was found in DBA subgroup. At the high viscosity group, microleakage of NB subgroup was higher than the two other subgroups, but Kruskal-Wallis test revealed no statistically significant difference between the three subgroups of low viscosity $(P=0.114)$ or high viscosity sealant groups $(P=0.648)$ [Table 1$]$.

\begin{tabular}{|c|c|c|c|c|c|c|}
\hline \multirow[t]{2}{*}{ Group } & \multirow[t]{2}{*}{$\begin{array}{l}\text { Sub } \\
\text { group* }\end{array}$} & \multicolumn{4}{|c|}{$\begin{array}{c}\text { Microleakage score } \\
\text { number }(\%)\end{array}$} & \multirow[t]{2}{*}{$P$ value } \\
\hline & & 0 & 1 & 2 & 3 & \\
\hline \multirow{3}{*}{$\begin{array}{l}\text { Low } \\
\text { viscosity } \\
\text { sealant }\end{array}$} & NB & $8(80)$ & $0(0)$ & $0(0)$ & $2(20)$ & \multirow[t]{3}{*}{0.114} \\
\hline & EBA & $6(60)$ & $3(30)$ & $0(0)$ & $1(10)$ & \\
\hline & DBA & $10(100)$ & $0(0)$ & $0(0)$ & $0(0)$ & \\
\hline \multirow{3}{*}{$\begin{array}{l}\text { High } \\
\text { viscosity } \\
\text { sealant }\end{array}$} & NB & $2(20)$ & $3(30)$ & $2(20)$ & $3(30)$ & \multirow[t]{3}{*}{0.648} \\
\hline & EBA & $4(40)$ & $1(10)$ & $1(10)$ & $4(40)$ & \\
\hline & DBA & $3(30)$ & $4(40)$ & $2(20)$ & $1(10)$ & \\
\hline
\end{tabular}

${ }^{*}$ NB: No bonding; EBA: Enamel bonding agent; DBA: Dentin bonding agent
The Mann-Whitney U-test showed a significant difference between the two viscosities in the NB subgroup $(P=0.041)$. Low viscosity sealant showed better marginal integrity than the other group [Figure1].

In the EBA subgroup, there was no significant difference between the two viscosities. (Mann-Whitney U-test, $P=0.171)$.

Comparison of the microleakage scores in the DBA subgroup showed a significant difference between the two viscosities (Mann-Whitney U-test, $P=0.002$ ). Microleakage at the low viscosity sealant group was significantly lesser than the high viscosity sealant group [Figure 2].

\section{DISCUSSION}

Use of fissure sealant after acid etching has been advocated as a reliable method for the prevention of fissure caries. There are several factors that may affect sealant success and among those are the effects of sealant viscosity and bonding agent application on marginal sealing. This study was based on a comparative assessment of microleakage of teeth sealed with high or low filled sealants with and without the application of bonding agents.

\section{Effect of bonding agent application}

The results indicate that in both high and low viscosity sealant groups, application of bonding and its type had no effect on microleakage. In the high viscosity sealant group, it was expected that using bonding (EBA or DBA) would result in better penetration and lower microleakage, but that was not the case. It seems that in spite of high viscosity, it still has enough flowability to penetrate into the microspaces and form resin tags. Kwon's study also showed that applying high viscosity sealant (60\% filled) without any bonding could result in resin tag formation and low microleakage. ${ }^{[18]}$ In an in vivo study, Boksman et al..$^{[19]}$ compared the retention rate of a filled and an unfilled sealant with and without using DBAs. After 2 years, they reported that using bonding agents did not increase the retention of either type of sealants.

However, in the low viscosity sealant group, it seems that the high flowability of the sealant is the main factor in reducing leakage and adding an intermediate layer of bonding agent has no additional effect on improving the marginal seal. It has been shown that the degree of penetration of a sealing agent into the interfacial micro-gap directly corresponds to the material's viscosity and potential to adequately wet 


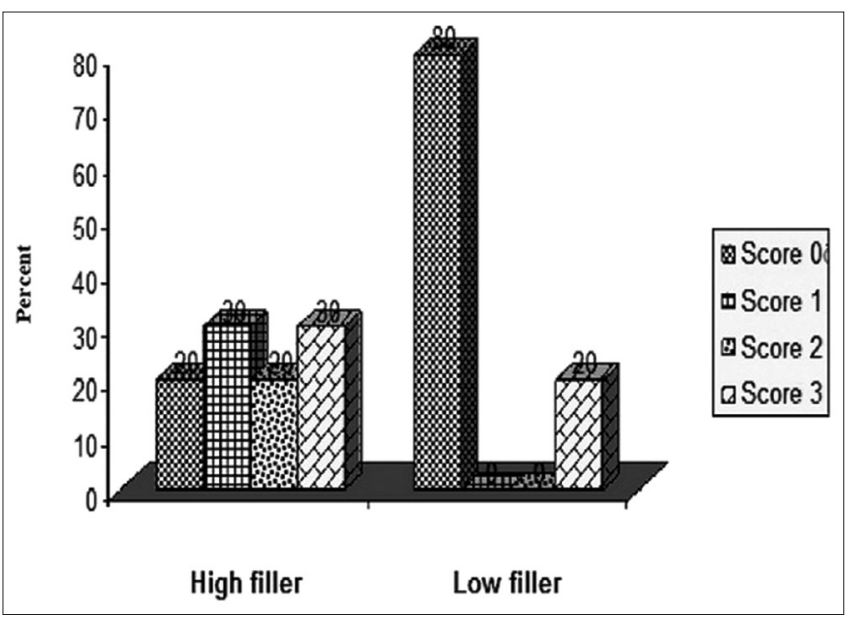

Figure 1: Distribution of microleakage scores in no bonding subgroup according to sealant viscosity

restoration or tooth surfaces. ${ }^{[20]}$ Percinoto et al. also mentioned that low viscose sealant had a greater potential to penetrate into the fissures and the micro porosities produced in the enamel by etching. ${ }^{[21]}$

A review article by Das and Suma ${ }^{[10]}$ showed that using bonding agent as an intermediary layer between enamel and sealant did not affect sealant success, except in cases in which adequate isolation and contamination control is impossible. As this was an in vitro study and there was no saliva contamination, it could be expected that the application of any bonding agent has no statistically significant effect on sealant microleakage. Also, other clinical studies ${ }^{[2,23]}$ revealed no significant differences with or without using bonding agents prior to sealant application. It seems that the success of a sealant is related to whether the sealant is applied under optimal conditions. The two latter studies have been performed in a dry and isolated situation, so the results are predictable.

\section{Effect of sealant viscosity}

The results showed that in the NB subgroup the low viscosity sealant had significantly lower microleakage. This result is in agreement with the results of other studies. Irinoda et al..$^{[13]}$ and Prabhakar et al. ${ }^{[24]}$ concluded that low viscosity sealant can penetrate fully into the etched surface and form a resin-infiltrated layer in enamel beyond the etched depth, whereas the high viscosity sealant cannot penetrate enough to provide good marginal seals. Kakaboura et al. reported that unfilled sealant penetrated deeper in narrow fissures than low viscosity resin composite and compomer. ${ }^{[25]}$ On the other hand, Barnes et al. ${ }^{[26]}$ and Droz et al., ${ }^{[27]}$ concluded that although the

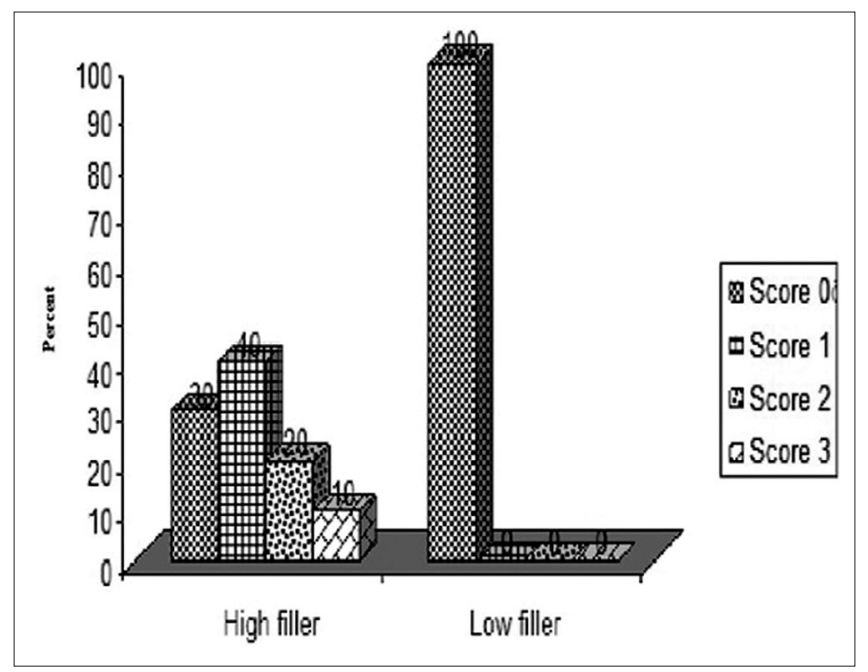

Figure 2: Distribution of microleakage scores in dentin bonding agent subgroup according to sealant viscosity

penetration of different commercial sealant products is different, viscosity and flow properties of a sealant have no effect on sealing capacity. This is parallel to the result of Breakspere and Wilton, which have shown that effective attachment of the sealant to enamel required to be brushed into the dry etched enamel and sealant viscosity seems unimportant. ${ }^{[28]}$ Other investigators such as Park et al. reported that unfilled and filled sealants exhibited no differences regarding the microleakage score. ${ }^{[16]}$

The low viscosity sealant also showed better results in the DBA subgroup, so that the combination of DBA and low viscosity sealant resulted in the samples not exhibiting any dye penetration. Better results in this subgroup than the NB subgroup could be partly explained based on the impact of the increased surface moisture-chasing effect of the hydrophilic primers (HEMA), increased flow as a result of the less viscous primer, and increased flexibility of polymerized bonding agent or sealant complex due to application of DBA. ${ }^{[29]}$

In the EBA subgroup, we expected the same result as the DBA subgroup, but there was no significant difference between the two sealants. The findings may be due to the fact that the EBA (Margin Bond ${ }^{\circledR}$ ) used in this study was an unfilled hydrophobic adhesive with lack of a primer to increase its wetting ability, and its fluidity was less than the used DBA. Hence, it seems that it leads to better results in the high viscosity sealant (the two samples with a score of 0 changed to 4 samples without microleakage as shown in Table 1). But, there were no good results in the low viscosity sealant group (the eight samples without 
microleakage changed to six samples with a score of 0 as shown in Table 1). Therefore, the dissimilarities between the two viscosities have been reduced so that there was no significant difference between the two sealants in the EBA subgroup.

Lack of access to a cutting machine with more delicate blades that would allow us to provide more slices per tooth for a more detailed microleakage assessment was one limitation in this study. Also, like other in vitro studies, the results need to be supported with clinical studies.

This study showed that low viscosity sealant was superior because it showed less microleakage when compared to the high viscosity sealant. However, the use of a bonding agent prior to the application of a pit and fissure sealant does not decrease the microleakage rate.

\section{CONCLUSIONS}

Based on the conditions in our study, the following conclusions were made:

- The Seal-Rite sealant with low filler particles acts better than Seal-Rite with high filler particles as a pit and fissure sealant material regarding microleakage

- The use of a bonding agent (DBA or EBA) prior to the application of a pit and fissure sealant does not decrease the microleakage rate.

\section{Acknowledgment}

This research was supported by the Vice Chancellor for research of the Mashhad University of Medical Sciences; this study was based on a thesis $(\mathrm{No}=2208)$ submitted to the Mashhad Dental School, Mashhad University of Medical Sciences (Iran), in partial fulfillment of the requirements for the Master of Science degree in Pediatric Dentistry.

\section{Financial support and sponsorship}

Nil.

\section{Conflicts of interest}

There are no conflicts of interest.

\section{REFERENCES}

1. Dye BA, Tan S, Smith V, Lewis BG, Barker LK, Thornton-Evans G, et al. Trends in oral health status: United States, 1988-1994 and 1999-2004. Vital Health Stat 11 2007;248:1-92.

2. Feigal RJ. Sealants and preventive restorations: Review of effectiveness and clinical changes for improvement. Pediatr Dent 1998;20:85-92.

3. Chaitra TR, Subba Reddy VV, Devarasa GM, Ravishankar TL. Flowable resin used as a sealant in molars using conventional, enameloplasty and fissurotomy techniques: An in vitro study. J Indian Soc Pedod Prev Dent 2010;28:145-50.

4. Mazhari F, Mehrabkhani M, Sadeghi S, Malekabadi KS. Effect of bevelling on marginal microleakage of buccal-surface fissure sealants in permanent teeth. Eur Arch Paediatr Dent 2009;10:241-3.

5. Borem LM, Feigal RJ. Reducing microleakage of sealants under salivary contamination: Digital-image analysis evaluation. Quintessence Int 1994;25:283-9.

6. Fritz UB, Finger WJ, Stean H. Salivary contamination during bonding procedures with a one-bottle adhesive system. Quintessence Int 1998;29:567-72.

7. Hebling J, Feigal RJ. Use of one-bottle adhesive as an intermediate bonding layer to reduce sealant microleakage on saliva-contaminated enamel. Am J Dent 2000;13:187-91.

8. Qadri GW, Noor SN, Mohamad D. Microleakage assessment of a repaired, nano-filled, resin-based fissure sealant. Pediatr Dent 2009;31:389-94.

9. Locker D, Jokovic A, Kay EJ. Prevention. Part 8: The use of pit and fissure sealants in preventing caries in the permanent dentition of children. Br Dent J 2003;195:375-8.

10. Das UM, Suma G. Bonding agents in pit and fissure sealants: A review. Int J Clin Pediatr Dent 2009;2:1-6.

11. Yamada Y, Hossain M, Shimizu Y, Kimura Y, Masuda Y, Nakamura $Y$, et al. Analysis of surface roughness and microleakage of fissure sealants following organic debris removal with Carisolv. J Dent 2008;36:130-7.

12. Dukic W, Dukic OL, Milardovic S. The influence of Healozone on microleakage and fissure penetration of different sealing materials. Coll Antropol 2009;33:157-62.

13. Irinoda $Y$, Matsumura $Y$, Kito H, Nakano T, Toyama T, Nakagaki H, et al. Effect of sealant viscosity on the penetration of resin into etched human enamel. Oper Dent 2000;25:274-82.

14. Papacchini F, Cury AH, Goracci C, Chieffi N, Tay FR, Polimeni A, et al. Noninvasive pit and fissure sealing: Microtensile bond strength to intact bovine enamel of different pit and fissure sealants in a simplified fissure model. J Adhes Dent 2006;8:375-80.

15. Papacchini F, Goracci C, Sadek FT, Monticelli F, Garcia-Godoy F, Ferrari M. Microtensile bond strength to ground enamel by glass-ionomers, resin-modified glass-ionomers, and resin composites used as pit and fissure sealants. J Dent 2005;33:459-67.

16. Park K, Georgescu M, Scherer W, Schulman A. Comparison of shear strength, fracture patterns, and microleakage among unfilled, filled, and fluoride-releasing sealants. Pediatr Dent 1993;15:418-21.

17. Montanari M, Pitzolu G, Felline C, Piana G. Marginal seal evaluation of different resin sealants used in pits and fissures. An in vitro study. Eur J Paediatr Dent 2008;9:125-31.

18. Kwon HB, Park KT. SEM and microleakage evaluation of 3 flowable composites as sealants without using bonding agents. Pediatr Dent 2006;28:48-53.

19. Boksman L, McConnell RJ, Carson B, McCutcheon-Jones EF. A 2-year clinical evaluation of two pit and fissure sealants placed with and without the use of a bonding agent. Quintessence Int 1993;24:131-3.

20. Owens BM, Johnson WW. Effect of new generation surface sealants on the marginal permeability of Class $\mathrm{V}$ resin composite restorations. Oper Dent 2006;31:481-8.

21. Percinoto C, Cunha RF, Delbem AC, Aragones A. Penetration of a light-cured glass ionomer and a resin sealant into occlusal fissures and etched enamel. Am J Dent 1995;8:20-2.

22. Jaberi Ansari Z, Hashemi SM. Effect of enamel bonding agents on pit and fissure sealant retention in an isolated situation. J Dent 2008;5:156-60.

23. Pinar A, Sepet E, Aren G, Bölükbasi N, Ulukapi H, Turan N. Clinical performance of sealants with and without a bonding agent. Quintessence Int 2005;36:355-60.

24. Prabhakar AR, Murthy SA, Sugandhan S. Comparative evaluation of the length of resin tags, viscosity and microleakage of pit and fissure sealants - An in vitro scanning electron microscope study. Contemp Clin Dent 2011;2:324-30.

25. Kakaboura A, Matthaiou L, Papagiannoulis L. In vitro study of penetration of flowable resin composite and compomer into occlusal fissures. Eur J Paediatr Dent 2002;3:205-9.

26. Barnes DM, Kihn P, von Fraunhofer JA, Elsabach A. Flow 
Mehrabkhani, et al.: Sealant, viscosity, and bonding

characteristics and sealing ability of fissure sealants. Oper Dent 2000;25:306-10.

27. Droz D, Schiele MJ, Panighi MM. Penetration and microleakage of dental sealants in artificial fissures. J Dent Child (Chic) 2004;71:41-4.

28. Breakspere RJ, Wilton A. Factors affecting the structure of a fissure sealant at the enamel/sealant interface. Aust Dent J 1977;22:199-202.

29. Feigal RJ, Donly KJ. The use of pit and fissure sealants. Pediatr Dent 2006;28:143-50.

\begin{tabular}{|l|l|}
\hline \multicolumn{2}{|c|}{ Access this article online } \\
\hline Quick Response Code: \\
\hline
\end{tabular}

